\title{
The CT and US features of Ewing's sarcoma/ primary neuroectodermal tumor of the kidney: two case reports and review of literature
}

This article was published in the following Dove Press journal:

OncoTargets and Therapy

17 March 2016

Number of times this article has been viewed

\section{Da-Fu Zhang* \\ Zhen-Hui Li* \\ De-Pei Gao \\ Guang-Jun Yang \\ Ying-Ying Ding \\ Guan-Shun Wang \\ Xing-Xiang Dong}

Department of Radiology, The Third Hospital of Kunming Medical University, Tumor Hospital of Yunnan Province, Kunming, People's Republic of China

*These authors contributed equally to this work
Correspondence: Ying-Ying Ding; De-Pei Gao

Department of Radiology, The Third Hospital of Kunming Medical University, Tumor Hospital of Yunnan Province, Kunzhou Road, Kunming 650II8,

People's Republic of China

Tel +86 I 388828 906I;

+8615969402258

Email d_yying@hotmail.com; gaodepei3II@sohu.com
Abstract: Ewing's sarcoma/primary neuroectodermal tumor (EWS/PNET) is an extraordinarily rare primary tumor of the kidney with characteristic histology. To date, the imaging features of EWS/PNET have not been clearly described. Here, we report two cases of EWS/PNET confirmed by fine-needle aspiration biopsy and analyze the findings of computed tomography and ultrasound. The radiological features of EWS/PNET are presented along with a brief review of the pertinent literature to have a further understanding of EWS/PNET's imaging features.

Keywords: Ewing's sarcoma, neuroectodermal tumor, X-ray computed tomography, histology, ultrasound

\section{Introduction}

Ewing's sarcoma/primary neuroectodermal tumor (EWS/PNET) most commonly arises from lower extremity (especially femur) and pelvis. However, EWS/PNET of the kidney is an extraordinally rare tumor that can be found in both children and adults. ${ }^{1,2}$ It was first described in 1974 by Seemayer et al. ${ }^{3}$ To date, only $\sim 126$ cases have been reported ${ }^{4-13}$ with very few detailed radiological descriptions. EWS/PNET of the kidney is not well recognized by either clinicians or radiologists, and the clinical and imaging features of EWS/PNET have not been well illustrated. We report two cases of renal EWS/PNET occurring in two men and analyze the findings of computed tomography (CT) and ultrasound (US).

\section{Case presentations}

Over the past 2 years, two cases of EWS/PNET of primary renal origin were diagnosed in our institutions confirmed by fine-needle aspiration biopsy.

\section{Case I}

A 19-year-old male (Figure 1) presented at our hospital with abdominal mass. Results of his blood chemistry and routine blood tests were within the normal range. Urine cytology showed no signs of malignancy, but the patient had an elevated CA-125 at $178 \mathrm{kU} / \mathrm{L}$. The abdominal CT and US findings were reviewed. The case underwent unenhanced and enhanced CT scans (arterial, venous, and excretory phases scanning) and coronal and sagittal multiplanar reconstructions in the venous phase. The unenhanced CT scan showed a unilateral large solid renal infiltrative mass completely replacing the right kidney (Figure 1A). The mass had a maximum diameter of $\sim 12 \mathrm{~cm}$. The margin of the mass was poorly defined with a lobulated contour. Tumor necrosis and hemorrhage were 

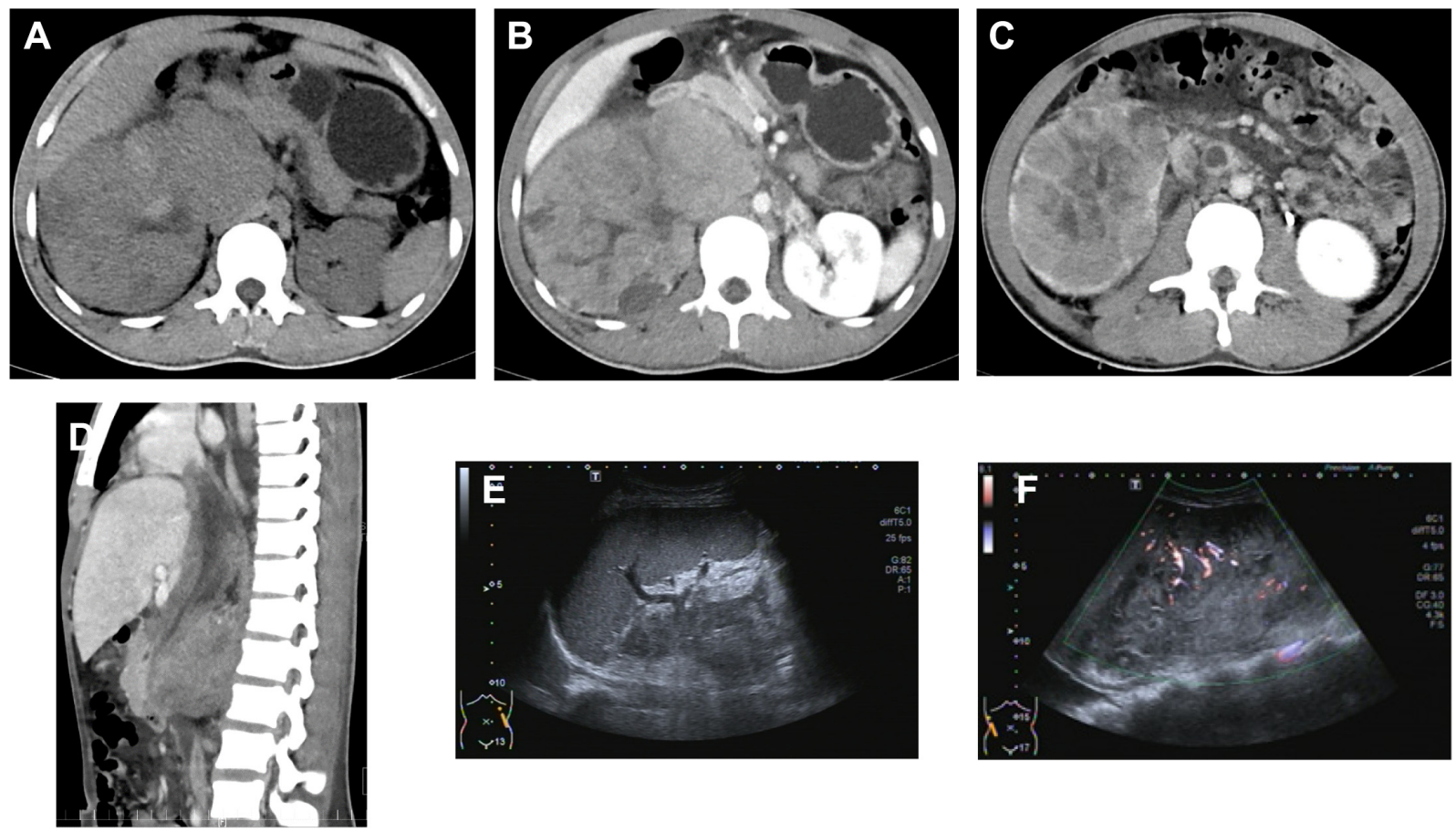

Figure I A renal EWS/PNET in a I9-year-old male with right flank pain.

Notes: (A) An unenhanced CT image shows a huge mass in the right kidney with heterogeneous attenuation. (B) A venous phase CT image shows heterogeneous subtle enhancement of the mass and a dilated left renal vein with thrombosis. (C) An excretory phase CT image shows multiple septum-like structures in the mass. (D) A sagittalreformatted image shows the lobulated contour of the mass and extension of the thrombosis in the IVC. Ultrasound (E) and Doppler (F) images show an ill-defined heterogenous hypoechoic mass with moderate vascularity in the right kidney with extension of the tumor into the IVC.

Abbreviations: CT, computed tomography; EWS/PNET, Ewing's sarcoma/primary neuroectodermal tumor; IVC, inferior vena cava.

detected in the case. The tumor showed weak or moderate heterogeneous enhancement (Figure 1B). The contrastenhanced CT showed that the tumor contained multiple irregular septa-like structures (Figure 1C). The patient had renal vein thrombosis and a metastasis to the regional lymph nodes. The tumor had an extension of thrombosis into the inferior vena cava and right atrium, presenting with lower extremity edema (Figure 1D). Extracapsular extension was detected in the case, but invasion to the adjacent organs was not detected. US showed an ill-defined, irregular, and slightly echogenic mass without acoustic shadowing, which replaced the right kidney completely (Figure $1 \mathrm{E}$ and F). The patient had renal vein thrombosis. Color Doppler flow imaging (CDFI) showed a moderate blood flow within the mass (Figure 1F). The patient did not undergo chemotherapy and surgery after a biopsy. The histopathology report revealed a small round blue cell tumor. On immunohistochemistry, the tumor cells stained positive for vimentin, CD99, Ki-67, Syn, and EWS rearrangement (EWS-FLI1 translocation) on fluorescent in situ hybridization.

\section{Case 2}

A 43-year-old male (Figure 2) presented at our hospital with abdominal pain and abdominal mass. Results of his blood chemistry and routine blood tests were within normal range. Urine cytology showed no signs of malignancy, but the patient had an elevated CA-125 at $345.7 \mathrm{kU} / \mathrm{L}$. The abdominal $\mathrm{CT}$ and US findings were reviewed. The case underwent unenhanced and enhanced CT scans (arterial, venous, and excretory phases scanning) and coronal and sagittal multiplanar reconstructions in the venous phase. The unenhanced CT scan showed a unilateral large solid renal infiltrative mass completely replacing the left kidney (Figure 2A). The mass had a maximum diameter of $\sim 12 \mathrm{~cm}$. The margin of the tumor was poorly defined with a lobulated contour. Tumor necrosis and hemorrhage were detected in the case. The mass showed weak or moderate heterogeneous enhancement. The contrast-enhanced CT showed that the tumor contained multiple irregular septa-like structures. The patients had renal vein thrombosis and a metastasis to the regional lymph nodes (Figure 2B and C). A lung metastasis was detected in the patient at the initial diagnosis (Figure 2D). Extracapsular extension was also detected in both, but invasion to the adjacent organs was not detected. US showed an ill-defined, irregular and slightly echogenic mass without acoustic shadowing, which replaced the left kidney completely. The patient had renal vein thrombosis and had extension of thrombosis into the inferior vena cava 

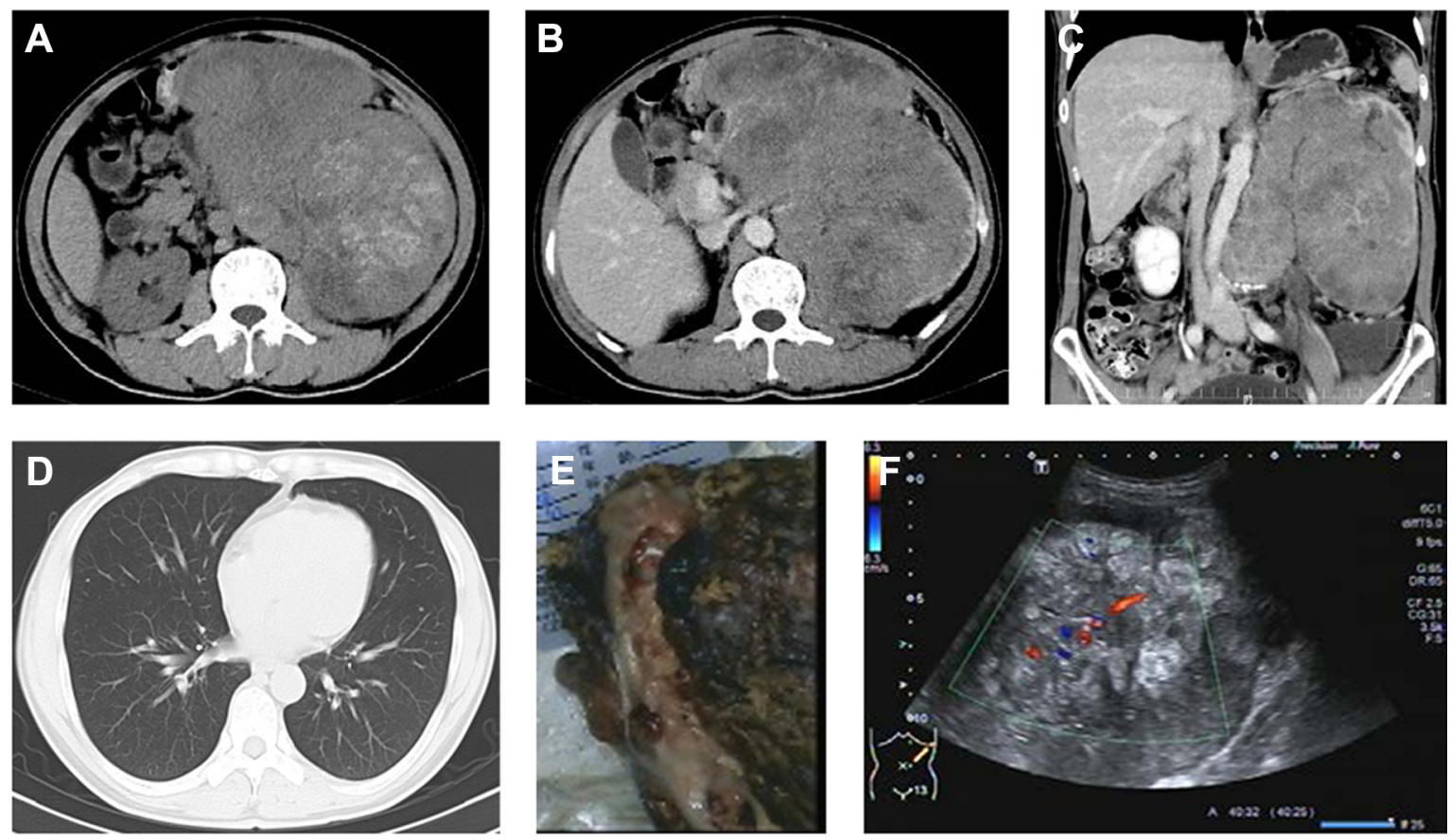

Figure 2 A 43-year-old male with abdominal mass.

Notes: (A) An unenhanced CT image shows a large mass in the left kidney with hemorrhage. (B) A contrast-enhanced CT image shows a thrombus in the left renal vein. (C) A sagittal-reformatted image shows the enlargement of the aortocaval lymph node compressing the artery. (D) A lung window setting shows the presence of metastatic lung nodules. (E) A photograph of a gross specimen shows a white and gray mass with hemorrhage and necrosis. (F) A Doppler image shows an ill-defined heterogenous hyperechoic mass with a little vascularity in the left kidney.

Abbreviation: $\mathrm{CT}$, computed tomography.

and right atrium. CDFI showed moderate blood flow within the mass (Figure 2F). The patient underwent chemotherapy after a biopsy; 2 months later, the size of the tumor decreased significantly, and the patient underwent a radical nephrectomy, but 3 months later, the patient had tumor recurrence including lung, liver, lymph node, and mesenteric metastases. The histopathology reports also revealed a small round blue cell tumor (Figure 3). On immunohistochemistry, the tumor cells stained positive for CD99 (Figure 4), CD117, Syn and
EWS rearrangement (EWS-FLI1 translocation) on fluorescent in situ hybridization.

\section{Discussion}

EWS/PNET of the kidney is an exceedingly rare entity, and only $\sim 126$ cases have been reported in literature. ${ }^{4-13}$ Due to the poor prognosis of primary renal EWS/PNET, accurate and timely diagnosis is demanded in patient diagnosis.

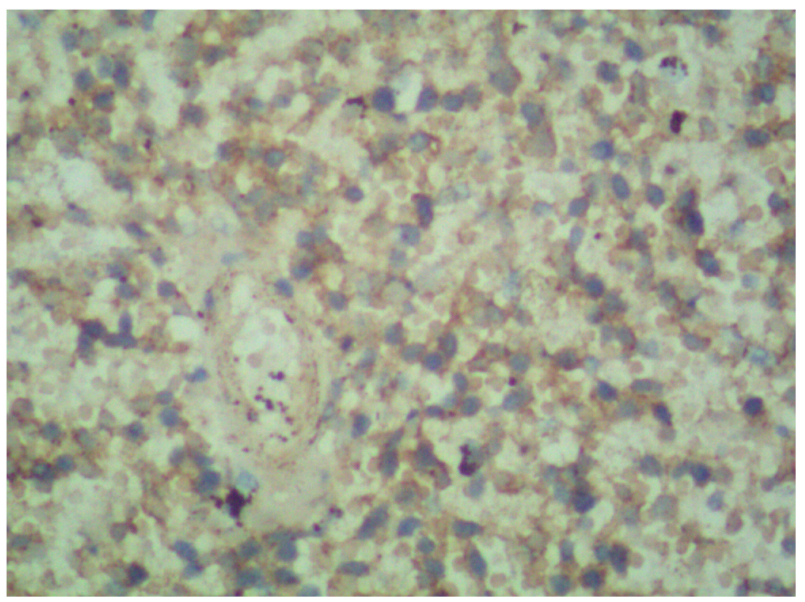

Figure 4 Strong membranous expression of CD99 (original magnification: $\times 200$ ).
Figure 3 Hematoxylin and eosin stain demonstrates a malignant, monotonous population of small round blue cells (original magnification: $\times 200$ ).

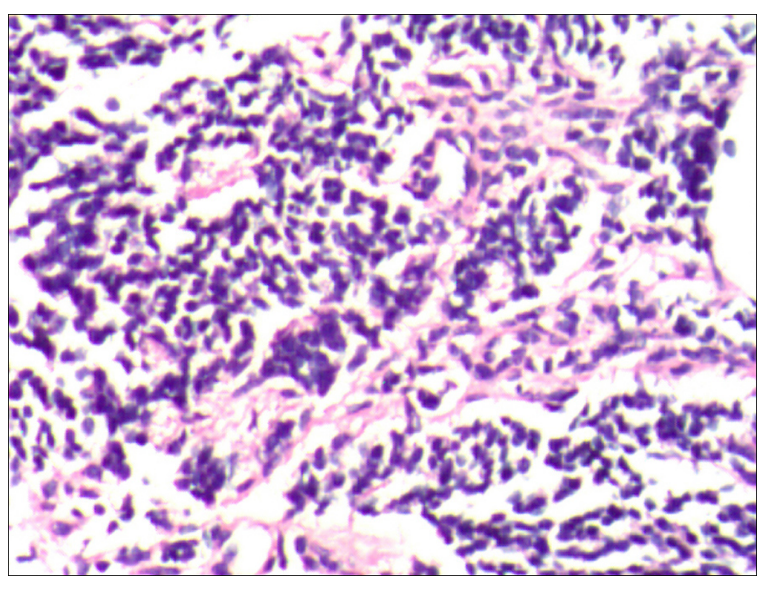

OncoTargets and Therapy 2016:9 
EWS/PNET of the kidney typically occurs in adolescents and young adults with a reported median age of 28 years with interquartile range (20-42 years), ${ }^{8}$ and the majority of them were male $(55 \%)$. The clinical presentation is variable and nonspecific with the complaint of a flank pain, palpable abdominal mass, or hematuria., ${ }^{8,14,15}$

Imaging findings in EWS/PNET are variable and nonspecific. The imaging characteristics demonstrate a significant overlap with other renal tumors, including renal cell carcinoma (RCC), Wilms's tumor, neuroblastoma, lymphoma, renal sarcomas, and metastatic carcinoma. ${ }^{16}$ The most common and representative imaging finding of a renal EWS/ PNET was characterized by a large mass with a heterogeneous contrast enhancement, necrotic or hemorrhagic areas and sometimes calcifications, and extensive venous thrombosis. On US, renal EWS/PNET may appear hyperechoic, isoechoic, and/or hypoechoic to the normal renal parenchyma and show an increased vascularity on $\mathrm{CDFI},{ }^{17}$ which is very similar to an advanced RCC. However, there are several characteristic imaging features of a renal EWS/PNET that differentiated the lesion from an advanced RCC. ${ }^{18}$

First, it is the presence of multiple irregular septum-like structures of the EWS/PNET. In our cases, multiple septumlike structures were detected (Figure 1C). Lee et a $\mathrm{l}^{18}$ found that all patients $(10 / 10)$ showed the septum-like structures. These septa were detected in both the necrotic and solid portions of the tumors, which were not frequently detected in advanced RCC. We also found similar enhancing septa on US (Figures $1 \mathrm{E}$ and $2 \mathrm{E}$ ).

Second, it is the difference in the pattern of the necrosis, hemorrhage, and cystic ingredient of the mass. ${ }^{5}$ Advanced RCCs usually present central necrosis or hemorrhage, whereas EWS/PNETs are characterized by the multifocal or pervasive necrosis and hemorrhage..$^{18}$ Both the tumors consisted of solid components with hemorrhagic, cystic, and necrotic areas, resulting in heterogeneous attenuation numbers on unenhanced and contrast-enhanced CT scans (Figures 1C and 2B) and heterogeneous echoic to the adjacent renal parenchyma on US.

Third, the EWS/PNET is characterized with low or moderate enhancement, in both the arterial and venous phase enhancement images. ${ }^{5,18}$ In our cases, low enhancement on the arterial phase and moderate enhancement on the venous phase were presented. Unlike large conventional RCCs, high enhancement on the arterial phase and low enhancement on the venous phase were presented. However, low enhancement cannot differentiate EWS/PNET from an unusual RCC, such as the papillary type and a collecting duct carcinoma.
Calcification was not presented in our cases. A study of ten patients found that only one patient showed foci of coarse calcification. ${ }^{18}$ Maybe the number of case was relatively small to analyze the true incidence of calcification.

Renal EWS/PNETs showed more aggressiveness when compared to conventional RCCs, and the majority of patients presented with advanced stage disease. EWS/PNETs also showed a high rate of venous thrombosis and the presence of distant metastases, including lymph node invasion, pulmonary metastases, liver metastases, and bone metastases $(25 \%-50 \%) .{ }^{19}$ Lee et al ${ }^{18}$ showed a high rate of renal vein thrombosis $(80 \%)$ and distant metastases $(40 \%)$. Risi et al ${ }^{8}$ reported that one-third of the patients were metastatic at diagnosis, and $40 \%$ of nonmetastatic patients had developed metastasis. In our cases, venous thrombosis and the presence of distant metastases were detected.

Although EWS/PNET of the kidney is very rare with nonspecific imaging findings, this tumor is typically characterized with a low enhanced large mass with multiple septumlike structures and peripheral hemorrhage. In addition, a renal EWS/PNET is an aggressive and rapidly progressing tumor that is usually detected in adolescents and young adults with the presence of venous thrombosis and distant metastasis. When the adolescents and young adults have a large renal mass with such characteristics, the EWS/PNET should be considered a diagnostic possibility.

\section{Acknowledgment}

This retrospective case series study was approved by the local ethics committee of the Third Hospital of Kunming Medical University, the Tumor Hospital of Yunnan Province, the Cancer Hospital of Yunnan Province, Kunming, Xishan Region, People's Republic of China, who waived the need for informed consent to be obtained because this case series is a retrospective study, and patients could not be contacted, as had been discharged from hospital.

\section{Disclosure}

The authors report no conflicts of interest in this work.

\section{References}

1. Balamuth NJ, Womer RB. Ewing's sarcoma. Lancet Oncol. 2010;11(2): 184-192.

2. Jimenez RE, Folpe AL, Lapham RL, et al. Primary Ewing's sarcoma/ primitive neuroectodermal tumor of the kidney: a clinicopathologic and immunohistochemical analysis of 11 cases. Am J Surg Pathol. 2002;26(3): 320-327.

3. Seemayer T, Thelmo W, Bolande R, Wiglesworth FW. Peripheral neuroectodermal tumors. Perspect Pediatr Pathol. 1975;2:151-172. 
4. Kumar R, Gautam U, Srinivasan R, et al. Primary Ewing's sarcoma/ primitive neuroectodermal tumor of the kidney: report of a case diagnosed by fine needle aspiration cytology and confirmed by immunocytochemistry and RT-PCR along with review of literature. Diagn Cytopathol. 2012; 40(Suppl 2):E156-E161.

5. De Visschere P, De Potter A, Claus F, et al. PNET/Ewing's sarcoma of the kidney: imaging findings in two cases. JBR-BTR. 2013;96(2): 75-77.

6. Yamamoto Y, Yamazaki K, Ishida Y. Upregulation of NKX2.2 of a target of EWSR1/FLI1fusion transcript, in primary renal Ewing sarcoma. J Cytol. 2015;32(1):30-32.

7. Faizan M, Anwar S, Iqbal S, et al. Primary renal Ewing's sarcoma: a rare entity. J Coll Physicians Surg Pak. 2014;24(Suppl 1):S66-S67.

8. Risi E, Iacovelli R, Altavilla A, et al. Clinical and pathological features of primary neuroectodermal tumor/Ewing sarcoma of the kidney. Urology. 2013;82(2):382-386.

9. Almeida MF, Patnana M, Korivi BR, Kalhor N, Marcal L. Ewing sarcoma of the kidney: a rare entity. Case Rep Radiol. 2014;2014:283902.

10. Liu Z, Wang X, Lu Y, Chen L, Lu Y. Primarywing sarcoma/primitive neuroectodermal tumor of the renal pelvis: a case report. World J Surg Oncol. 2014;12:293.

11. Chakrabarti N, Dewasi N, Das S, Bandyopadhyay A, Bhaduri N. Primary Ewing's sarcoma/primitive neuroectodermal tumor of kidney-a diagnostic dilemma. Iran J Cancer Prev. 2015;8(2):129-133.
12. Pakravan A, Vo TM, Sandomirsky M, Bastani B. Primary Ewing sarcoma of kidney in an elderly. Iran J Kidney Dis. 2012;6(4):307-310.

13. Kakkar S, Gupta D, Kaur G, Rana V. Primary primitive neuroectodermal tumor of kidney: a rare case report with diagnostic challenge. Indian $J$ Pathol Microbiol. 2014;57(2):298-300.

14. Karpate A, Menon S, Basak R, Yuvaraja TB, Tongaonkar HB, Desai SB Ewing sarcoma/primitive neuroectodermal tumor of the kidney: clinicopathologic analysis of 34 cases. Ann Diagn Pathol. 2012;16(4):267-274.

15. Thyavihally YB, Tongaonkar HB, Gupta S, et al. Primitive neuroectodermal tumor of the kidney: a single institute series of 16 patients. Urology. 2008;71(2):292-296.

16. Lalwani N, Prasad SR, Vikram R, Katabathina V, Shanbhogue A, Restrepo C. Pediatric and adult primary sarcomas of the kidney: a cross-sectional imaging review. Acta Radiol. 2011;52(4):448-457.

17. Katkar AS, Vinu-nair S, Savage J, Chintapalli K. Primary renal Ewing sarcoma/primitive neuroectodermal tumor (PNET) of the kidney, can it be diagnosed on imaging? a case report. Austin J Radiol. 2014;1(1):4.

18. Lee H, Cho JY, Kim SH, Jung DC, Kim JK, Choi HJ. Imaging findings of frimitive neuroectodermal tumors of the kidney. J Comput Assist Tomogr. 2009;33(6):882-886.

19. Hari S, Jain T, Thulkar S, Bakhshi S. Imaging features of feripheral primitive neuroectodermal tumours. Br J Radiol. 2008;81(972):975-983.
OncoTargets and Therapy

\section{Publish your work in this journal}

OncoTargets and Therapy is an international, peer-reviewed, open access journal focusing on the pathological basis of all cancers, potential targets for therapy and treatment protocols employed to improve the management of cancer patients. The journal also focuses on the impact of management programs and new therapeutic agents and protocols on

\section{Dovepress}

patient perspectives such as quality of life, adherence and satisfaction. The manuscript management system is completely online and includes a very quick and fair peer-review system, which is all easy to use. Visit http://www.dovepress.com/testimonials.php to read real quotes from published authors. 